\title{
The increasing demand and necessity for a team approach
}

\author{
M. K. Ross ${ }^{1}$
}

\begin{abstract}
The role of professionals complementary to dentistry (PCDs) has changed radically in recent times and further changes are likely in the foreseeable future. The modernisation of dentistry throughout the UK dictates that the existing workforce takes into account the substantial contribution which can be made by PCDs in addressing the unacceptable levels of disease which exist in many parts of the country.
\end{abstract}

Both dental hygienists and therapists have quite recently been given increased clinical responsibilities. In addition to this, the new generation of dually qualified hygienisttherapists, or oral health practitioners, which may be an appropriate designation, heralds the emergence of a group of professionals who will be able to undertake much of the routine dentistry amongst both the child and adult population. Given the apparent shortage of dentists throughout the UK, these highly skilled individuals will have a significant contribution to make to the oral healthcare of the population. Recent investigations in Scotland have revealed that much ignorance exists about the clinical remit of this group and indeed, a degree of suspicion prevails about their ability to undertake procedures which historically have been in the domain of the dentist. Additionally, we are fast-approaching mandatory continuing professional development for the entire dental team, and our research demonstrates quite clearly that significant problems exist in terms of access, funding and remuneration for PCDs to undertake this.

Although these advances in clinical input by PCDs have been long-awaited and ultimately well-received, consideration should be given to other progress that

\footnotetext{
1*Senior Lecturer, Edinburgh Postgraduate Dental Institute, 4th Floor Lauriston Building, Lauriston Place, Edinburgh EH3 9HA

${ }^{*}$ Correspondence to: Margaret K. Ross

Email: Margaret.K.Ross@ed.ac.uk
}

This article highlights the paper being presented at 13.30 on Thursday, 6 May 2004 in the Tregonwell Hall at The British Dental Conference \&t Exhibition 2004 being held at the Bournemouth International Centre

could be made in the future. In comparison with medicine, dentistry has lacked vision in terms of patient care. Medicine has developed to the extent of now having nurse specialists and consultants and indeed, in more remote and rural areas of the country, much of routine medical care is undertaken by individuals who have received appropriate training. Only recently, an announcement was made where general nurses will be permitted to undertake certain procedures in the field of plastic surgery. These people work in tandem with other medical professionals who all have expertise in their own fields. Surely the same situation could exist in dentistry where individuals have extended roles and are complementary to each other?

To achieve this, further changes are necessary and should be considered now so that another lengthy time period does not elapse for progression to be made. The need for hygienists, therapists and oral health practitioners to work from a written prescription is a hindrance, as is the need for a dentist to be on the premises whilst certain procedures are being undertaken. The benefits of being able to administer inferior dental block anaesthesia to a patient are obvious, but the need for a dentist to be on-site is less clear. To provide clinically skilled individuals who have received appropriate training with prescribing abilities would also be a welcome and appropriate advancement, particularly for those who may be employed in remote and rural areas. This could also be said of the need to be able to provide emergency care for patients with oral problems which, as the situation stands at the moment, cannot be undertaken without this often nebulous referral system.

The role of dental nurses could be expanded significantly to allow them, again following appropriate training, to undertake more clinically involved procedures in line with nurses in medicine. Examples of these tasks could be in relation to the taking of impressions, suture removal, placement of temporary restorations and triage duties, to name but a few. Although triage dental nurses do exist in the UK, it is thought that they are not being used to their full potential.

The overall delivery of dentistry is destined to change, and this presentation will attempt to discuss the way forward in relation to more appropriate use of the existing workforce. Do we really need more dentists to treat oral disease?

The British Dental Conference \&t Exhibition 2004 is being held at the Bournemouth International Centre between Thursday 6 th and Saturday 8th May 2004

Contact: DMS (Delegate Management Services) for further information:

Tel: 08701666625 or +44 (0) 1252771425

Fax: 08705228890 or +44 (0) 1252771790

For the latest update on the agenda and to download the programme visit: www.bda-events.org 